\title{
Postpartum Infectious Sacroiliitis Temporally-Related to Con- tinuous Labor Epidural Analgesia: Was the Epidural to Blame? A Case Report and Review of the Literature

\author{
Mafdy Basta, MD*
}

Department of Anesthesiology and Perioperative Medicine, Medical College of Georgia at Augusta University, USA

*Corresponding author: Mafdy Basta, MD, Assistant Professor, Department of Anesthesiology and Perioperative Medicine, Medical College of Georgia at Augusta University, 1120 15 ${ }^{\text {th }}$ St., Augusta, GA 30912, USA, Tel: (706)721-3871, Fax: (706)721-7753

\begin{abstract}
Infectious (bacterial) sacroiliitis, though rare, yet needs to be considered at the differential diagnosis of back/leg pain in parturients. The following is a case of infectious sacroiliitis in a postpartum patient that presented four days after delivery with hip and leg pain. Interestingly, the patient's labor analgesia was conducted with a continuous lumbar epidural analgesia; however, a causal relationship remains to be determined.
\end{abstract}

\section{Keywords}

Pregnancy, Labor epidural, Infectious sacroiliitis

\section{List of Abbreviations}

WBCs: White Blood Cells; ED: Emergency Department; LE: Lower Extremity; SI: Sacroiliac; MRI: Magnetic Resonance Imaging; ISI: Infectious Sacroiliitis; CT: Computerized Tomography; ESR: Erythrocyte Sedimentation Rate; CRP: C Reactive Protein

\section{Case Report}

The patient is a 41-year-old gravida 2 para 2 African American female that underwent elective induction of labor at 39 weeks gestation due to chronic hypertension. The patient's labor analgesia was conducted with continuous lumbar epidural that was described as uneventful. Spontaneous vaginal delivery occurred about nineteen hours after the epidural placement. The patient did not receive antibiotics prior to delivery as isolation of group B streptococcus by endocervix swab two weeks prior was reported as negative, though low level colonization could not be excluded with a negative test. The patient was afebrile and her White Blood Cells (WBCs) count was reported within normal range prior to delivery. The patient's labor was complicated by small right-sided periurethral and left-sided vaginal lacerations that were repaired at the bedside by the obstetric team. Following delivery, the epidural catheter was removed with tip intact and site was noted to be clean and dry.

On postpartum day 1 , the patient started to complain of sharp pain in the left hip and gluteal regions with radiation down the left leg that, after evaluation, was attributed to possible entrapment neuropathy encountered during labor. The patient was reassured and treated conservatively with nonsteroidal anti-inflammatory drugs and physical therapy. The patient was discharged home on postpartum day 2 after some improvement at her hip and leg pain and instructed to follow up as scheduled.

On postpartum day 4 , the patient presented to the Emergency Department (ED) with worsening left hip and left leg pain that persisted since her vaginal delivery. The patient described the pain as sharp, radiating down the entire left lower extremity (LE), and made worse by movement. The pain was severe enough so as to interfere with her getting out of bed. Occasional scattered numbness and tingling along the left LE were also reported. No fever reported. No abnormal bladder or bowel symptoms reported. 




Figure 1: Fat saturated T1-weighted axial image showing asymmetric fluid distention of left sacroiliac joint (blue arrow).

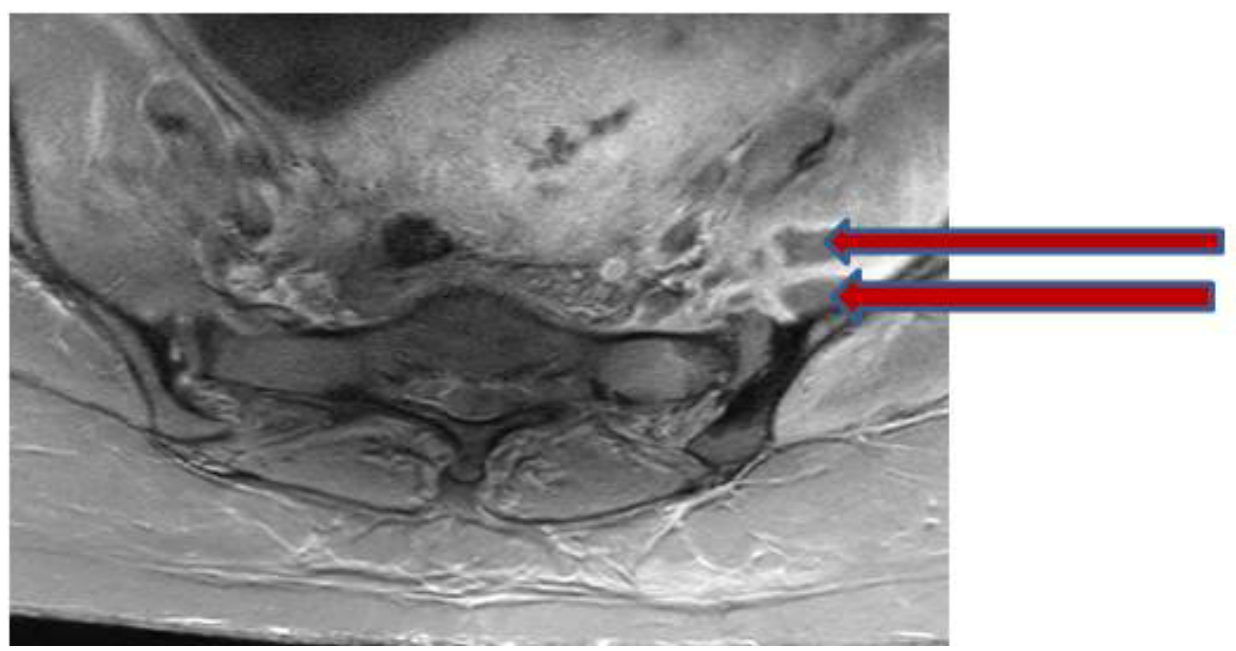

Figure 2: Fat saturated T1-weighted axial image demonstrates peripherally enhancing fluid collections within the left iliopsoas muscle (red arrows).

Relevant laboratory findings at ED visit revealed polynuclear neutrophil leukocytosis. The patient was admitted to the obstetric team for further management.

In light of recent epidural catheter placement, an urgent magnetic resonance imaging (MRI) of the lumbar spine with contrast enhancement was ordered. No evidence of epidural hematoma or abscess was present. However, incidental findings of asymmetric fluid distention of the left sacroiliac (SI) joint (Figure 1) with fluid collections within the left iliopsoas, left piriformis, and left gluteus muscles (Figure 2) were detected, findings concerning for infectious/inflammatory sacroiliitis (ISI). Neuroradiology consult was placed, and under computerized tomography (CT) guidance, about $10 \mathrm{ml}$ of purulent fluid was aspirated from left SI joint, and sent for Gram stain and culture. Infectious Disease consult was placed and the patient was started on empiric vanco- mycin pending culture results. Further work up included measurement of erythrocyte sedimentation rate (ESR), and C-reactive protein (CRP), both were found to be about three fold elevated. Blood cultures were drawn but reported later as negative for any growth. Gram stain of SI joint fluid revealed Gram positive cocci that on culture were identified as group B streptococci (Strep. agalactiae). Vancomycin was changed to ceftriaxone per sensitivity results. An orthopedic consult for septic arthritis was also placed, but conservative management was recommended in light of clinical (decreasing pain), and laboratory (resolving leukocytosis, decreasing ESR and (RP) improvement. The patient was discharged home on long term (6 weeks) intravenous antibiotics and recommended to follow up as instructed.

\section{Discussion}

The prevalence of infectious sacroiliitis (ISI) is rare, 
involving between one and two percent of septic arthritis cases, which is probably due to the poor vascularization of this joint, resulting in a low risk of infection via the hematogenous route [1,2]. The most frequent clinical finding is lumbogluteal pain, whereas coxofemoral pain, pubic pain, and abdominal pain could also be present [3]. The heterogeneity of clinical signs associated with ISI may be accounted for by the anatomical structure of the SI joint. The first and second sacral roots pass near the SI joints, with the joint capsule being bordered by the psoas muscle in front and the gluteal and pyriformis muscles behind. Depending on the capsular region involved in the arthritis, the pain may be gluteal or inguinal, mimicking damage to the hip. Involvement of the anterior joint capsule causes peritoneal irritation, and painful abdominal symptoms. Age-related changes to the SI joint, such as decreased vascularization and mobility that limit the risk of bacterial colonization, may explain the low frequency of ISI in elderly individuals and its increased incidence in children [4].

The greater demands on the SI joint in pregnant women due to increased weight and hormone-induced changes to the pelvic conformation account for the higher frequency of ISI cases during pregnancy, and in the immediate post-partum period [5-7].

ISI has been described in the literature after lumbar epidural analgesia, although a causal relationship remains unclear [8,9]. It has been suggested that the pathogenesis of ISI is hematogenous bacterial spread. Whether the insertion of an epidural catheter during bacteremia can causally contribute to ISI is uncertain, though its association has been proposed [8,9].

Edelstein, et al. described a patient who developed ISI five days after normal vaginal delivery in the absence of other potential risk factors other than epidural insertion [8].

In the case presented by Mulvey, a patient developed ISI ten days after emergency cesarean section [9]. Risk factors for the latter included artificial rupture of membranes and operative delivery.

Generally, the infection is unilateral, with a preference for the left side [10]. Risk factors in ISI cases include intravenous drug use [11], pelvic trauma, infectious endocarditis, hemoglobinopathy, immunosuppressive treatment, as well as cutaneous, respiratory or genitourinary infections. Polynuclear neutrophil leukocytosis in addition to increased levels of CRP and ESR are standard features of ISI.

The presence of fever is variable. In pregnant women, fever may be absent in up to one third of cases [5].

$\mathrm{MRI}$ is the imaging study of choice for establishing the diagnosis of ISI and enables to assess whether the infection has spread to adjacent muscular structures [12]. When a microorganism is identified, Gram positive Staphylococci are the predominate pathogens.
Gram-negative bacilli most frequently identified are Salmonella species, encountered more frequently in children than adults [13]. Pseudomonas aeruginosa are most commonly encountered in immunosuppressed patients, hospitalized patients, and intravenous drug users [11]. Streptococcus is more frequent agent when functional gynecological signs are present $[5,6]$. Brucella is a frequent cause of septic arthritis in certain parts of the world, such as Turkey [14].

Even though the majority of pregnancy-associated ISI cases reported were due to pyogenic bacteria other than group B streptococci [2], ISI due to group B streptococci has been described before [10].

Definitive microbiological diagnosis may be based on blood cultures, joint fluid by CT-guided percutaneous puncture, or surgical investigations [15].

In the case presented here, though no definite conclusions could be reached, yet isolation of group $B$ streptococci, a frequent colonizer of the female genital tract, favors hematogenous and/or local spread during the labor process as the likely culprit mechanism for infection.

The bacterial spread was likely enhanced by the presence of periurethral and vaginal tears. Low level genital colonization with group B streptococci could have been present and explains the negative endocervix swab result obtained two weeks prior to labor. The lack of localized skin infection at the site of epidural insertion and the absence of an epidural abscess on subsequent MRI substantially decreases the likelihood of the epidural catheter as being a culprit in this case. It is also possible that ISI was present prior to the epidural analgesia, although the lack of back and/or leg pain at initial hospital admission would argue against that.

In the absence of any identified microorganism, it is preferable to consider antibiotic therapy active against Staphylococcus and Gram-negative bacilli, administered parenterally for two weeks followed by oral treatment for six weeks [16].

\section{Conclusion}

Postpartum infectious sacroiliitis, though uncommon, yet needs to be included at the differential diagnosis of back/leg pain. Early diagnosis by imaging studies and prompt treatment with antibiotics can help control the infection and prevent long term morbidity. Whether a causal relationship between epidural analgesia and ISI exists could not be determined with any degree of certainty and needs further studies.

\section{References}

1. Mancarella L, De Santis M, Magarelli N, leradi AM, Bonomo L, et al. (2009) Septic sacroiliitis: An uncommon septic arthritis. Clin Exp Rheumatol 27: 1004-1008.

2. Vyskocil JJ, Mcllroy MA, Brennan TA, Wilson FM (1991) 
Pyogenic infection of the sacroiliac joint. Case reports and review of the literature. Medicine (Baltimore) 70: 188-197.

3. Zrig M, Mnif H, Zrig A, Koubaa M, Jawahdou R, et al. (2010) Iliopsoas abscess: a rare complication of pyogenic sacroiliitis in a child. Arch Pediatr 17: 141-143.

4. Taylor ZW, Ryan DD, Ross LA (2010) Increased incidence of sacroiliac joint infection at a children's hospital. J Pediatr Orthop 30: 893-898.

5. Almoujahed MO, Khatib R, Baran J (2003) Pregnancy-associated pyogenic sacroiliitis: Case report and review. Infect Dis Obstet Gynecol 11: 53-57.

6. Yansouni CP, Ponette V, Rouleau D (2009) Bacterial sacroiliitis and gluteal abscess after dilation and curettage for incomplete abortion. Obstet Gynecol 114: 440-443.

7. Moros ML, Rodrigo C, Villacampa A, Ruiz J, Lapresta C (2009) Septic shock in pregnancy due to pyogenic sacroiliitis: A case report. J Med Case Reports 3: 6505.

8. Edelstein S, Edoute $Y(2003)$ Bacterial sacroiliitis probably induced by lumbar epidural analgesia. Infect Dis Obstet Gynecol 11: 105-108.

9. Mulvey JM (2008) Postpartum septic sacroiliitis coincident with labour epidural analgesia. Anaesth Intensive Care 36: 875-878.
10. M Jedwab, S Ovadia, M Dan (1999) Pyogenic sacroiliitis in pregnancy. Int J Gsynaecol Obstet 65: 303-304.

11. Ferraro K, Cohen MA (2004) Acute septic sacroiliitis in an injection drug user. Am J Emerg Med 22: 60-61.

12. Stürzenbecher A, Braun J, Paris S, Biedermann T, Hamm B, et al. (2000) MR imaging of septic sacroiliitis. Skeletal Radiol 29: 439-446.

13. Garg B, Madan M, Kumar V, Malhotra R (2011) Sacroiliitis caused by Salmonella typhi: A case report. J Orthop Surg (Hong Kong) 19: 244-246.

14. Turan $H$, Serefhanoglu K, Karadeli E, Togan T, Arslan $H$ (2011) Osteoarticular involvement among 202 brucellosis cases identified in Central Anatolia region of Turkey. Intern Med 50: 421-428.

15. Chan DSY, Saklani A, Shah PR, Haray PN (2010) Laparoscopic drainage of retroperitoneal abscess secondary to pyogenic sacroiliitis. Ann R Coll Surg Engl 92: 32-34.

16. Roblot F, Besnier JM, Juhel L, Vidal C, Ragot S, et al. (2007) Optimal duration of antibiotic therapy in vertebral osteomyelitis. Semin Arthritis Rheum 36: 269-277. 